\title{
List of abbreviations used in the present volume 1 Grammatical codes
}

\begin{tabular}{ll}
1 & 1st person \\
2 & 2nd person \\
3 & 3rd person \\
ABS & absolutive \\
ACC & accusative \\
ADJ & adjective \\
ADV & adverb \\
ADVR & adverbializer \\
ANTIP & antipassive \\
AOR & aorist \\
ART & article \\
AUG & augment \\
AUX & auxiliary \\
BEN & benefactive \\
CAUS & causative \\
CL & clitic \\
CLF & classifier \\
CLIT & clitic \\
CNG & co-negative \\
COM & comitative \\
COMP & complement/complementizer \\
COMPL & completive \\
COND & conditional \\
CONJ & conjunction \\
CONN & connective \\
DAT & dative \\
DEF & definite \\
DEICT & deictic \\
DEM & demonstrative \\
DEO & deontic \\
DERIV & derivative \\
DET & determiner \\
DIM & diminutive \\
DIREC & directional \\
DISC & discontinuative \\
DIST & distal \\
DYN & dynamic \\
ELAT & elative \\
EM & epistemic marker \\
EMPH & emphatic \\
EPEN & epenthetic \\
ERG & ergative \\
& \\
\hline DON
\end{tabular}




\begin{tabular}{ll} 
EVID & evidential \\
EXCL & exclusive \\
EXIST & existential \\
F & feminine, female \\
FEM & feminine \\
FILL & filler \\
FOC & focus \\
FUT & future \\
FUT.IMP & future imperative \\
GEN & genitive \\
GM & generalizing modality marker \\
HON & honorific \\
HORT & hortative \\
ILL & illative \\
IMP & imperative \\
IMPRS & impersonal \\
INCOMPL & incompletive \\
IND & indicative \\
INDEF & indefinite \\
INDF & indefinite \\
INF & infinitive \\
INF1 & 1st infinitive \\
INF2 & 2nd infinitive \\
INF3 & 3rd infinitive \\
INFL & inflectional \\
INS & instrumental \\
INTENS & intensifier \\
INTERJ & interjection \\
INTR & intransitive \\
IPFV & imperfective \\
IRR & irrealis \\
LEX & lexical \\
LOC & locative \\
M & masculine, male \\
MASC & masculine \\
MDL & modal \\
MP & mediopassive \\
MV & modal verb \\
N & neuter \\
NEC & necessitative \\
NEG & negation/negative/negator \\
NEUT & neuter \\
NFIN & non-finite \\
NML & nominal \\
NMLZ & nominalizer \\
NOM & nominative \\
NONMDL & non-modal \\
& \\
\hline
\end{tabular}




$\begin{array}{ll}\text { NONPST } & \text { non-past } \\ \text { N.PROP } & \text { proper noun } \\ \text { OBJ } & \text { object } \\ \text { OBLIG } & \text { obligation } \\ \text { ONOM } & \text { onomatopoetic } \\ \text { OPT } & \text { optative } \\ \text { OSTEN } & \text { ostensive } \\ \text { PARTIT } & \text { partitive } \\ \text { PASS } & \text { passive } \\ \text { PFV } & \text { perfective } \\ \text { PL } & \text { plural } \\ \text { POL } & \text { polite } \\ \text { POSS } & \text { possessive } \\ \text { PP } & \text { past participle } \\ \text { PREF } & \text { prefix } \\ \text { PREP } & \text { preposition } \\ \text { PRO } & \text { pronoun } \\ \text { PROG } & \text { progressive } \\ \text { PRS } & \text { present } \\ \text { PSB } & \text { possibility } \\ \text { PST } & \text { past } \\ \text { PTCP } & \text { participle } \\ \text { PTL } & \text { particle } \\ \text { Q } & \text { question } \\ \text { QUOT } & \text { quotative } \\ \text { REFL } & \text { reflexive } \\ \text { REPORT } & \text { reportative } \\ \text { RI } & \text { root infinitive } \\ \text { SBJ } & \text { subject } \\ \text { SBJV } & \text { subjunctive } \\ \text { SE } & \text { sentence-ending } \\ \text { SG } & \text { singular } \\ \text { SOV } & \text { subject-object-verb } \\ \text { STAT } & \text { stative } \\ \text { SUBJ } & \text { subjunctive } \\ \text { SUP } & \text { supinum } \\ \text { TAM } & \text { tense-aspect-mood/modality } \\ \text { TOP } & \text { topic } \\ \text { TOPS } & \text { topic shift } \\ \text { TR } & \text { transitive } \\ \text { TRANSL } & \text { translative } \\ \text { V } & \text { verb } \\ \text { VOC } & \text { vocative } \\ & \end{array}$




\section{Other abbreviations}

$\begin{array}{ll}\text { ADS } & \text { adult-directed speech } \\ \text { CDS } & \text { child-directed speech } \\ \text { CHAT } & \text { Codes for the Human Analysis of Transcripts } \\ \text { CHI } & \text { child, children } \\ \text { CHILDES } & \text { Child Language Data Exchange System } \\ \text { CLAN } & \text { Computerized Language Analysis } \\ \text { CS } & \text { child speech } \\ \text { DP } & \text { data point } \\ \text { FAT } & \text { father } \\ \text { HSES } & \text { high socio-economic status } \\ \text { ID } & \text { identifier } \\ \text { IQ } & \text { intelligence quotient } \\ \text { ISCED } & \text { International Standard Classification of Education } \\ \text { ISEI } & \text { International Socio-economic Index of Occupational Status } \\ \text { LSES } & \text { low socio-economic status } \\ \text { MLU } & \text { mean length of utterance } \\ \text { MOT } & \text { mother } \\ \text { MS } & \text { Microsoft } \\ \text { OECD } & \text { Organization for Economic Co-operation and Development } \\ \text { P } & \text { probability of rejecting the null hypothesis } \\ \text { PAR } & \text { parent(s) } \\ \text { RG } & \text { Russian Grammar } \\ \text { SAP } & \text { speech act participant } \\ \text { SE } & \text { standard error } \\ \text { SES } & \text { socio-economic status } \\ \text { TOK } & \text { tokens } \\ \text { TYP } & \text { types } \\ & \end{array}$

\section{Symbols}

${ }^{*} 0 \quad$ missing inflection

[//] retracing

$=\quad$ clitic boundary

$\beta \quad$ beta value, estimate of fixed effect 